\title{
Resolución de problemas en el foro virtual
}

\author{
Sandra Lucía Guerra Gómez
}

\begin{abstract}
Resumen
El presente documento presenta parte de los resultados de una investigación realizada con estudiantes, de la modalidad a distancia, del programa de Licenciatura en Educación Básica de la Universidad Pedagógica y Tecnológica de Colombia (UPTC), cuyo objetivo buscó describir y analizar cómo se desarrolló el aprendizaje a través de resolución de problemas en el foro virtual. Para esto se realizó la investigación basada en un estudio de caso, donde se analizó la información obtenida de la aplicación de una estrategia en un ambiente diferente a las aplicadas en encuentros presenciales y en la que, finalmente, se describió el proceso de aprendizaje en los estudiantes seleccionados para tal fin.
\end{abstract}

En la investigación se tomó como base las fuentes primarias que surgieron de la obtención de información de los participantes a través de sus intervenciones en un ambiente virtual. Como fuentes secundarias, unas bases mínimas estructuradas desde tres perspectivas de revisión: concepciones sobre aprendizaje, estrategia de resolución de problemas, abordada desde el concepto de problema, resolución de problema y la enseñanza de la estrategia de resolución de problemas, $y$, finalmente, la metodología de educación a distancia con el apoyo de ambientes virtuales de aprendizaje.

Con la aplicación de la estrategia de "Resolución de Problemas"; se promovió un aprendizaje consciente, comprometido, disciplinado, donde el estudiante aprendió a aprender, aprendió activamente, realizó mejoras continuas de su proceso, lo que evidenció con ello, mayor compromiso con los procesos de aprendizaje.

Palabras clave: aprendizaje, foro virtual, problemas ambientales, resolución de problemas.

1 Licenciada en Ciencias de la Educación Biología y Química1998 - UPTC. Especialista en manejo Biológico de Cultivos 2002 - UPTC. Magister en Educación 2011 - UPTC. Docente ocasional Tiempo Completo Universidad Pedagógica y Tecnológica de Colombia. Facultad de Estudios a Distancia. Integrante del grupo de investigación SIEK (Saberes interdisciplinarios en construcción). Correo electrónico: guerrasandralucia@yahoo.es 


\title{
Solving problems in the virtual forum
}

\begin{abstract}
This paper presents part of the research results conducted with basic education students of the distance learning program from Universidad Pedagógica Tecnológica de Colombia (UPTC). The main goal was to describe and analyze how learning through problem solving in the virtual forum. This study was based on a case study where was analyzed information obtained from the application of different strategy that employed in face-to-face meetings, describing the student learning process.
\end{abstract}

This study was based on primary sources meaning information obtained from participants interventions in virtual environment. As secondary sources were used minimum bases from three reviewed structured perspectives:, conceptions of learning strategy, troubleshooting approached from the problem concept, problem solving and teaching problem solving strategy, and methodology of distance education supported by virtual learning environments.

With the implementation of "Problem Solving" strategy was promoted a conscious committed and disciplined learning. Where students learned how to learn, learned actively, and in improve continuously their learning process, showing a greater commitment with learning processes.

Key words: Characterization, environmental problems, earning, troubleshooting, virtual forum. 


\section{Marco teórico}

El entorno establece factores y condiciones que potencian o dificultan los procesos de aprendizaje de las personas. Los escenarios de aprendizaje, las exigencias del ambiente educativo, las necesidades propias de las personas, la disponibilidad y variedad de la tecnología, los ambientes virtuales de aprendizaje, todos forman parte de un complejo en el que se debe decidir, aceptar, proponer, disponer, para quien aprende, a su vez, produzca y conviva en el sentido que perfecciona su aprendizaje.

Por lo anterior, el aprendizaje es considerado como un proceso que cumple un papel predominante en el ser humano, desde el cual la persona aprende a manejar información, a construir conocimiento y a desarrollar competencias que le permitirán vivir con conciencia en el mundo y con una orientación hacia el fortalecimiento de su propio desarrollo.

Argüelles (2007), considera el aprendizaje como un proceso social mediado por el cambio, que implica la construcción activa de sentido, a través de los viejos y nuevos conocimientos, así como conocimientos acerca de cómo aprender. Esto exige motivación y se encuentra influido por el concepto de sí mismo que tiene quien aprende; a su vez implica un pensamiento orientado hacia metas, organizado, constructivo y estratégico.

El aprendizaje, como todas las actividades humanas, se fundamenta en una serie de procedimientos y acciones que posibilitan la apropiación, comprensión e integración de conocimientos a la estructura cognoscitiva de la persona. Tal como lo menciona Vos Savant (1998), el dominio del proceso de aprendizaje: "Permitirá la consecución del control efectivo sobre los procesos mentales, desarrollando instrumentos intelectuales para resolver problemas complejos con eficiencia".

De otra parte, para llevar a cabo la estrategia de resolución de problemas, en esta investigación, se basó en la propuesta de enseñanza referida por Gil et al. (1989), quienes afirman que es un modelo que permite que los estudiantes desarrollen procesos adecuados cuando se enfrentan a problemas y/o ejercicios cerrados; donde se ven favorecidos aspectos relacionados con la metacognición, que mejora la situación del aprendizaje escolar. A continuación se mencionan los aspectos que cobija esta estrategia:

- Plantear situaciones problémicas: deben generar interés teniendo en cuenta destrezas y actitudes de los estudiantes.

-Estudio cualitativo de las situaciones problémicas: los estudiantes comienzan a estructurar un plan para la resolución del problema. 
-Orientar el tratamiento científico: formulación de hipótesis, elaboración de estrategias que contrasten dichas hipótesis, además de las estrategias propias para realizar el análisis de los resultados, donde se tengan en cuenta predicciones de las hipótesis generadas por el grupo de estudiantes o por la comunidad.

- Plantear el manejo de nuevos conocimientos: tarea que requiere profundización de los conocimientos y consolidación de los mismos, en especial en las relaciones ciencia, tecnología y sociedad, que de una u otra manera, dan rigurosidad y carácter que tiene todo proceso científico.

-Actividades de síntesis: se plasman los resultados a través de la elaboración de productos que refuerzan el interés por el proceso y la concepción de nuevos problemas, para favorecer la metacognición.

Para implementar dicha estrategia, se decidió hacer uso de un ambiente virtual y, en consecuencia, a lo referido por Gutiérrez y Prieto (1996), sobre el conocimiento que el docente debe tener sobre el apoyo de las tecnologías en la mediación pedagógica; además de "Pensar en educar y promover el desarrollo de competencias comunicativas acordes con el entorno comunicativo e infocomunicacional de la sociedad contemporánea..." Huergo (1999). Es oportuno hacer referencia al Foro virtual como la herramienta utilizada para generar discusiones y del cual se obtuvo información valiosa para la investigación.

El foro virtual es concebido como un espacio de discusión sobre temas, en particular, que concentra opiniones de muchas personas de distintos lugares. Hay intercambio de mensajes entre los estudiantes y entre éstos y el profesor, permite la superación de las limitaciones espacio-temporales, y facilita la lectura, el debate y la opinión, lo que fomenta la comunicación, el aprendizaje cooperativo y la cohesión del grupo. Al foro virtual puede entrar cualquier persona que tenga alguna duda sobre un tema en especial, de manera que pueda compartir con un grupo sus inquietudes al respecto.

Cabero (1999), sobre un foro virtual dice: "Mejora la calidad y efectividad de la interacción, apoyando procesos de aprendizaje colaborativo, la participación activa y la interacción de todos frente a modelos más tradicionales de aprendizaje", de tal manera que es muy útil para encontrar soluciones a problemas, porque permite que varias personas den su opinión y a la vez, ayuden a dar respuesta a la pregunta inicialmente planteada.

Por las particularidades de los participantes de esta investigación, se utilizó el foro para promover las discusiones, teniendo en cuenta que los estudiantes provienen de diferentes contextos o regiones. Esta es una característica del 
foro virtual, pues el intercambio de opiniones, los debates, las experiencias compartidas, las dudas y, en especial, la resolución de problemas, permiten que el foro virtual sea de gran utilidad didáctica.

\section{Metodología}

Se optó por el estudio de caso para la investigación realizada, por cuanto permitió examinar un sistema definido o un caso en detalle, donde se emplearon fuentes de datos encontrados en el entorno (Yin, 1994). Además, porque se dió lugar a la comprensión del aprendizaje que se generó en los estudiantes, al establecimiento de unos criterios de evaluación que determinaron dicho proceso, que permitieron a su vez el análisis del fenómeno, entendiendo cómo, desde estas experiencias, este se construyó y se validó.

A través del diseño de un instrumento de evaluación, que permitió describir la aplicación de la estrategia de resolución de problemas basado en los criterios establecidos por Gil (1989) y los resultados de la misma en un ambiente virtual, se definió el diseño de un instrumento (matriz de evaluación) con unas proposiciones teóricas, para determinar la información correspondiente en cada una de ellas.

Según lo establecido por Yin (1994), una unidad de análisis dada es la manera como se dió el aprendizaje a través de resolución de problemas, además, se tomó en cuenta las proposiciones establecidas según los criterios de Gil (1989), y un instrumento para registrar, a través de puntuaciones, los aspectos alcanzados por los estudiantes. Después se realizó la trascripción de los aportes de los participantes de la investigación con el fin de tomar aspectos importantes y claves para el análisis de los datos, que a su vez permitieron caracterizar el aprendizaje en los estudiantes y presentar los resultados del análisis.

\section{Resultados de la investigación}

Del análisis, resultado de la investigación, surgieron unas características sobre la manera como se desarrolló el aprendizaje en los estudiantes participantes en la investigación, dentro de las que se destacaron: el aprendizaje se centró en el estudiante, el aprendizaje fue activo, se comprobó que los estudiantes realizaran mejoras continuas, el estudiante se comprometió en el hacer en lugar de únicamente aprender sobre algo; y el estudiante realizó un producto. Como evidencia de su aprendizaje se describen a continuación: 


\section{-EI aprendizaje se centró en el estudiante}

Se evidenció la motivación y los intereses en dedicar tiempo y esfuerzo en la resolución de un problema propuesto. Hubo preocupación por la construcción de nuevos conocimientos y habilidades en medio de un ambiente virtual asincrónico. Los participantes realizaron indagaciones a través de múltiples fuentes de información, tales como Internet, libros y sus propias experiencias, entre otros. Además realizaron la autoevaluación, aprendiendo de esta manera a evaluar su propio trabajo y a autorregular sus conocimientos.

Lo anterior se sustenta con lo referido por Pavón (2005), quien señala que el aprendizaje viene a partir de la actividad cognitiva que realice el estudiante con la información, la estructura didáctica en la cual el docente lo inserta y las demandas cognitivas que se le pide que haga con el material. Es decir, se logró a través de la motivación realizada en el estudiante, centrar su atención en la resolución de un problema, se dió un aprendizaje donde el estudiante se apropió del mundo y de sí mismo, y como manifiestan Gutiérrez y Prieto (1996), fueron dueños de los instrumentos necesarios para expresarse y comunicarse solidariamente, en la que resolvieron problemas de tipo ambiental que aquejan a la sociedad. Es lo que Gil refiere como "generación de interés” en las cuales los estudiantes muestran destrezas y actitudes frente a su propio proceso de aprendizaje.

\section{-El aprendizaje fue activo}

Hace referencia a que los participantes aprendieron a aprender el uno del otro y también la forma de ayudar a que sus compañeros aprendieran. Así mismo, evaluaron el trabajo y realizaron retroalimentación constructiva tanto para ellos mismos como para sus compañeros.

Edith Litwin (2005), señala que para adaptarse a los desarrollos tecnológicos, el hombre debe tener capacidad para identificar y desplegar actividades cognitivas nuevas, en tanto, las tecnologías permanentemente van generando distintas posibilidades para fortalecer el aprendizaje. Esto se complementa con la utilización de materiales que contienen toda la información, estructura, secuencia y elementos de retroalimentación para aprender un contenido de modo significativo (Barberá 2004; Barberá \& Rochera 2008). En este espacio, se buscó abrir el camino a nuevas relaciones del estudiante con el propio contexto, con la información obtenida, con sus compañeros de aprendizaje incluido el docente, consigo mismo y con su futuro. De igual manera, se propendió básicamente, porque los estudiantes tuvieran la capacidad de solucionar problemas, lo cual implicó en ellos tomar decisiones, adaptar soluciones, usar la imaginación y habilidades personales y sociales que les permitieran crecer como personas responsables, autónomas, sociables. 


\section{-Mejoras continuas}

Esto se manifestó en la medida que los estudiantes se tomaron el tiempo necesario para realizar intervenciones, lograron además aprender a tomar las decisiones necesarias para alcanzar un adecuado nivel de aprendizaje, se despertó el espíritu de indagación de los participantes al relacionar sus conocimientos con las situaciones cotidianas de su medio ambiente y social. Tal como lo plantea Gil et al. (1991), esa construcción nunca se propone para cuestionar ideas, para provocar cambios conceptuales, sino para resolver problemas de interés que se abordan, a partir de los conocimientos que tienen los participantes y de nuevas ideas que se construyen a título tentativo. En ese proceso, las concepciones iniciales experimentaron cambios e incluso, se cuestionaron radicalmente, aunque el objetivo fuera la resolución de los problemas presentados.

Lo anterior se sustenta con base en la afirmación realizada por Gutiérrez y Prieto (1996), al referir que toda práctica de resolución de problemas está orientada siempre hacia el futuro, toda vez que ella significa el diagnóstico, la comprensión, y la toma de decisión entre más de una alternativa. Por lo tanto, en los estudiantes se observó una actitud activa para resolver un problema, donde se evidenció la capacidad de relacionar los conocimientos adquiridos y la preocupación de buscar otros nuevos.

Ante lo propuesto por Gil, se logró plantear el manejo de nuevos conocimientos, siendo una tarea que requiere profundización de los conocimientos y consolidación de los mismos, en especial en las relaciones ciencia, tecnología $y$ sociedad que de una u otra manera, dan rigurosidad y carácter que tiene todo proceso científico. Esto se explica porque los estudiantes demostraron el manejo de nuevas ideas a partir de las propuestas realizadas en sus aportes y con los cuales permitieron que sus compañeros ofrecieran recursos con los cuales se logra procesos de reflexión frente a sus intervenciones.

\section{-Hacer más que aprender sobre algo}

En este aspecto se observó el compromiso de los participantes y la necesidad de comunicarse, compartir y ayudarse en el ambiente de aprendizaje, en el que se le suministró retroalimentación en cada caso. Los estudiantes aprendieron a depender más de ellos mismos y a aclarar las cosas por sí mismos.

De igual manera, se identifica con lo referido por Gutiérrez y Prieto (1996), en un hacer conceptual que respondió al desarrollo de capacidades como el pensar, tomar decisiones frente a la manera como debían resolver el problema. Se evidenció un hacer discursivo que como lo manifiesta el mismo autor corresponde a que los estudiantes desarrollaron capacidades para expresarse 
y comunicarse en el foro virtual y, en especial, el aprendizaje se centró en un hacer aplicativo pues los estudiantes desarrollaron el proceso de investigar para resolver el problema citado.

Teniendo como base el desarrollo de la estrategia de resolución de problemas, a través una ambiente virtual y, particularmente, el valor pedagógico de éstos, se encuentra la posibilidad y ventaja que ofrece discutir alrededor de diferentes contenidos en los mismos, pues en el contexto educativo el solo hecho de discutir, es de por sí una estrategia efectiva de aprendizaje, toda vez que es necesario articular y comunicar ideas, así como, escuchar y valorar las ideas de los otros (Argüelles, 2007).

\section{-Realización de un producto}

En cuanto a la realización de un producto, este incluyó el diseño y la presentación de un resultado que otros pudieran ver o conocer a través de las interacciones. Frente a lo anterior, Gil refiere dentro de la estrategia de resolución de problemas las actividades de sintesis como aquellas en las cuales se plasman los resultados, a través de la elaboración de productos que refuerzan el interés por el proceso y la concepción de nuevos problemas. Esto favorece la metacognición.

Este aspecto cobró un valor importante ya que ofreció una información complementaria y particular en cada uno de los estudiantes. Permitió además que ellos aprendieran de sus errores, los enfrentaran y superaran, convirtiéndose en retos difíciles e inesperados en un ambiente virtual. Esto confirma lo mencionado por Prendes (2003), quien considera que en los procesos de aprendizaje en un entorno virtual se trata de alumnos que trabajan en grupo e intercambian ideas, se hacen preguntas, todos escuchan y comprenden las respuestas, se ayudan entre ellos antes de pedir ayuda al profesor y, finalmente, obtienen un único producto del trabajo de grupo.

Así mismo se menciona dentro del presente análisis que se encontraron elementos que dejan ver cómo desde las intervenciones realizadas en el foro, el estudiante es protagonista de su proceso de aprendizaje, en el cual fortalecieron sus conocimientos a través de la estrategia implementada donde realizaron procesos de investigación, reflexión y gestión de su aprendizaje, transfiriendo el conocimiento a cualquier contexto. Este factor resultó determinante y además proyectivo en lo que ha llegado a ser hoy, el aprendizaje en ambientes virtuales. Se permitió identificar a través de sus participaciones y de manera explícitas la forma como se aprende a través de una estrategia como lo es la resolución de problemas.

Por lo tanto, como primer elemento, es que el aprendizaje se desarrolló a partir de la exigencia de los estudiantes en ir más allá de la información dada, elemento 
que evidencia que el estudiante lo realiza con el propósito de crear algo nuevo reconfigurado. En este proceso expanden y aplican los conocimientos previos y construyen con ese conocimiento; este elemento brinda tanto al investigador como a docentes en formación, la oportunidad de constatar el desarrollo de la comprensión de una temática a lo largo del tiempo y en situaciones nuevas para el aprendizaje. De igual manera, como lo menciona Salinas (2003), se trata en todo caso, de procesos de aprendizaje colaborativo, ya que se logra un contexto que enfatiza en la interacción en grupo donde los estudiantes participaron autónomamente en su proceso de aprendizaje, mientras resolvían un problema como grupo.

De otra parte, cabe resaltar que otro elemento importante de este proceso de aprendizaje y que contribuyó significativamente al mismo, fue la evaluación. Esta promovió la comprensión del proceso de aprendizaje y fué algo más que un examen al final de una unidad. Estas valoraciones informaron a los estudiantes y al docente las comprensiones que se han alcanzado y cómo proceder en la enseñanza y el aprendizaje posteriores. Por esto, la importancia de diseñar los instrumentos de evaluación del aprendizaje, y darlas a conocer a los estudiantes antes de que procedan a demostrar el conocimiento alcanzado.

Luego de conocer los criterios de evaluación sobre la estrategia de resolución de problemas, los participantes realizaron reflexiones sobre su proceso, indicando con ello una de las cualidades del aprendizaje como lo es la autorregulación del aprendizaje. En efecto, se identificó en las participaciones realizadas que los docentes se apropiaron paulatinamente de las instrucciones, ideas, estructuras, conocimiento, prácticas que estaban fuera de su pensamiento. Esta apropiación del conocimiento, donde se alcanzó con el dominio autónomo de lo aprendido, permitió identificar los procesos metacognitivos del estudiante para fortalecer su aprendizaje que muestran la manera como se aprende.

Se encontró además que los estudiantes plantearon situaciones problema teniendo en cuenta las ideas, destrezas y actitudes, lo que despertó el interés por el desarrollo de las actividades; este elemento permitió la toma de decisiones para acotar el problema propuesto, donde los estudiantes comenzaron a concebir un plan para su solución; orientaron la respuesta científico del problema, e incluyeron la invención de conceptos y elaboración de estrategias de intervención en el aula, analizando con coherencia el cuerpo de conocimientos. Plantearon el manejo de nuevos conocimientos en diversas situaciones para profundizar y afianzar los mismos, enfatizaron en la relación Ciencia/Tecnología/Sociedad, enmarcada en el desarrollo científico. Además las actividades de síntesis (donde realizaron la presentación de sus aportes de manera creativa) y la elaboración del resultado final, rompieron con el planteamiento de tareas escolares que permitieron a su vez la concepción de nuevos problemas que pueden ser solucionados en su propio contexto. 
Otro elemento encontrado fue la autogestión del aprendizaje, donde los estudiantes, como bien lo expresa Dewey, se educaron y se formaron en cuanto a la oportunidad para reflexionar críticamente sobre lo que hicieron. Esta reflexión crítica, según Rolheiser (2000), conllevó el desarrollo de la metacognición, al desarrollo del pensamiento crítico, al desarrollo de habilidades de solución de problemas, a la toma de decisiones y al desarrollo de un autoconcepto. Por lo tanto, se infiere que ellos interiorizaron estas operaciones mentales que le facilitaron las herramientas para la autogestión eficiente de su proceso de aprendizaje.

Frente a esta descripción de lo sucedido con las participaciones de los estudiantes al resolver un problema ambiental de manera espontánea y sin la mediación del tutor, se puede decir que es importante y necesario para que el aprendizaje sea significativo, que a su vez es básico para que exista la capacidad de dar sentido a lo que se aprende y, en consecuencia, como lo menciona Gutiérrez y Prieto (1996), la actitud activa ante una nueva situación que pide creatividad, capacidad de relacionar los conocimientos adquiridos y de buscar otros nuevos. Lo anterior, por cuanto se hizo necesario que el tutor investigador entrara a mediar el proceso y poner en conocimiento los criterios por tener en cuenta para la resolución de problemas, no para evaluar conocimientos, sino para promover el aprendizaje y a la vez fomentar la capacidad de autogestión del conocimiento, de tal manera que los estudiantes al conocer el instrumento dado, realizaran apropiación de sus ideas y las dieran a conocer a través de sus aportes.

\section{Conclusiones}

Finalmente, estas intervenciones permitieron concluir que los estudiantes internalizaron el proceso, es decir, se apropiaron paulatinamente de las instrucciones, ideas, estructuras, conocimiento y prácticas que estaban fuera de su pensamiento, y que esta internalización o apropiación del conocimiento, se alcanzó con el dominio autónomo de lo aprendido. Además se evidenció la interiorización en el momento en el que los estudiantes, de manera responsable, realizaron la autorregulación de su proceso, después de concretarse la estructura del ambiente de aprendizaje y adentrarse en los procesos requeridos para la obtención de resultados satisfactorios en su proceso de aprendizaje.

Con la aplicación de la estrategia resolución de problemas se logró promover el aprendizaje en los docentes, con la cual se les indicó cómo enfrentarse a problemas de su entorno y en el aula; se motivaron sobre una forma de pensar y actuar ante situaciones que se pueden resolver sin incertidumbre. Se demostró que no se enseña a resolver problemas, sino que se muestra los diferentes caminos que se pueden tomar para dar soluciones a problemas reales, según los conocimientos que se tienen de las temáticas identificadas. 


\section{Revista de}

investigaciones UNAD

Volumen 12. Número 1. Junio 2013

Se desarrolló el aprendizaje a través de los aportes realizados por los participantes, la resolución de dudas, donde se promovió además la discusión y motivando a los participantes, de tal manera que se aseguró el fortalecimiento de los procesos de aprendizaje. 


\section{Referencias bibliográficas}

Argüelles, D y Nofal, N. (2007). Estrategias para promover procesos de aprendizaje autónomo. Colombia: Alfaomega.

Barberá, E. (2004). La educación en red. Actividades virtuales de enseñanza y aprendizaje. España: Paidós.

Barberá, E, y M.J Rochera. (2008). Los entornos virtuales de aprendizaje basados en el diseño de materiales autosuficientes y el aprendizaje autodirigido. En Psicología de la educación virtual, editado por C. Coll y C. Monereo. España: Morata.

Gálvis Panqueva, A. (2008). Criterios y rúbrica TIGRE para autocontrolar calidad de aportes en discusiones. Disponible en http://aportetigre.blogspot.com/

Gil, D., Dumasumas-Carré, A., Caillot, M., Martínez Torregrosa, J. \& Ramírez, L. (1989). La Resolución de Problemas de lápiz y papel como actividad de investigación. Investigación en la escuela, 6, pp. 3-20.

Gil Pérez, D. (1991). La metodología científica y la enseñanza de las ciencias naturales Relaciones controvertidas. Enseñanza de las ciencias 4(2), 1986. Reimpreso en planteamientos pedagógicos, 1(2). 38-60.

Gutiérrez, F y Prieto D. (1996). Mediación pedagógica, Universidad de San Carlos de Guatemala, Guatemala.

Hernández, F. (1993). Concepciones en el estudio del aprendizaje de los estudiantes universitarios. Revista de investigación educativa, 22(2), 117-150.

Hernández S, R.; Fernández C.; Baptista, L. (2007). Metodología de la Investigación: (4 ed.). Mexico: Mc Graw Hill.

Huergo, J y Fernández, M. (1999). Cultura escolar, cultura mediática/intersecciones, Bogotá: Universidad pedagógica Nacional.

Litwin, Edith (Comp.). (2005). La educación a distancia. Temas para el debate en una nueva agenda educativa, Buenos Aires, Amorrortu, 160 págs., pág. 15.

Martínez, P. (2006). El método de estudio de caso, estrategia metodológica de la investigación científica. En: Pensamiento y gestión, $\mathrm{N}^{\circ}$ 20, Universidad del Norte. Pág. 165 - 193.

Pavón, F. (2005). Entornos Virtuales de enseñanza y aprendizaje (EVEA). En: Tecnologías de la información y comunicación para la formación de docentes. Madrid: Ediciones Pirámide. 


\section{Revistade}

Prendes, M. (2003). Trabajo colaborativo en espacios virtuales. En Cabero, J., Martínez, F. \& Salinas, J. (Coor.) Medios y herramientas de comunicación para la educación universitaria. Ciudad de Panamá: EDUTEC.

Rolheiser, C; Bower, B. y Stevahn, L. (2000). The portfolio organizer: succeeding with portfolios in your classroom. Association for supervision and curriculum development (ASCD). Canadá. [Libro en línea]. Disponible http://www.ascd.org/readingroom/ books/rolheiseroobook.html

Salinas, J. (2003). El diseño de procesos de aprendizaje cooperativo en situaciones virtuales. En Martínez Sánchez, F (compilador), Redes de comunicación en la enseñanza. Las nuevas perspectivas del trabajo corporativo. Barcelona: Paidós.

Vos Savant, Marilin. (1998). Gimnasia mental. Bogotá: Círculo de Lectores.

Yin, R. K. (1989). Case Study Research: Design and Methods, Applied Social Research Methods Series, Newbury Park CA, Sage.

......... (1994). Case Study Research - Design and Methods, Applied Social Research Methods, Vol. 5, 2nd. Edition. Newbury Park, CA, Sage. 


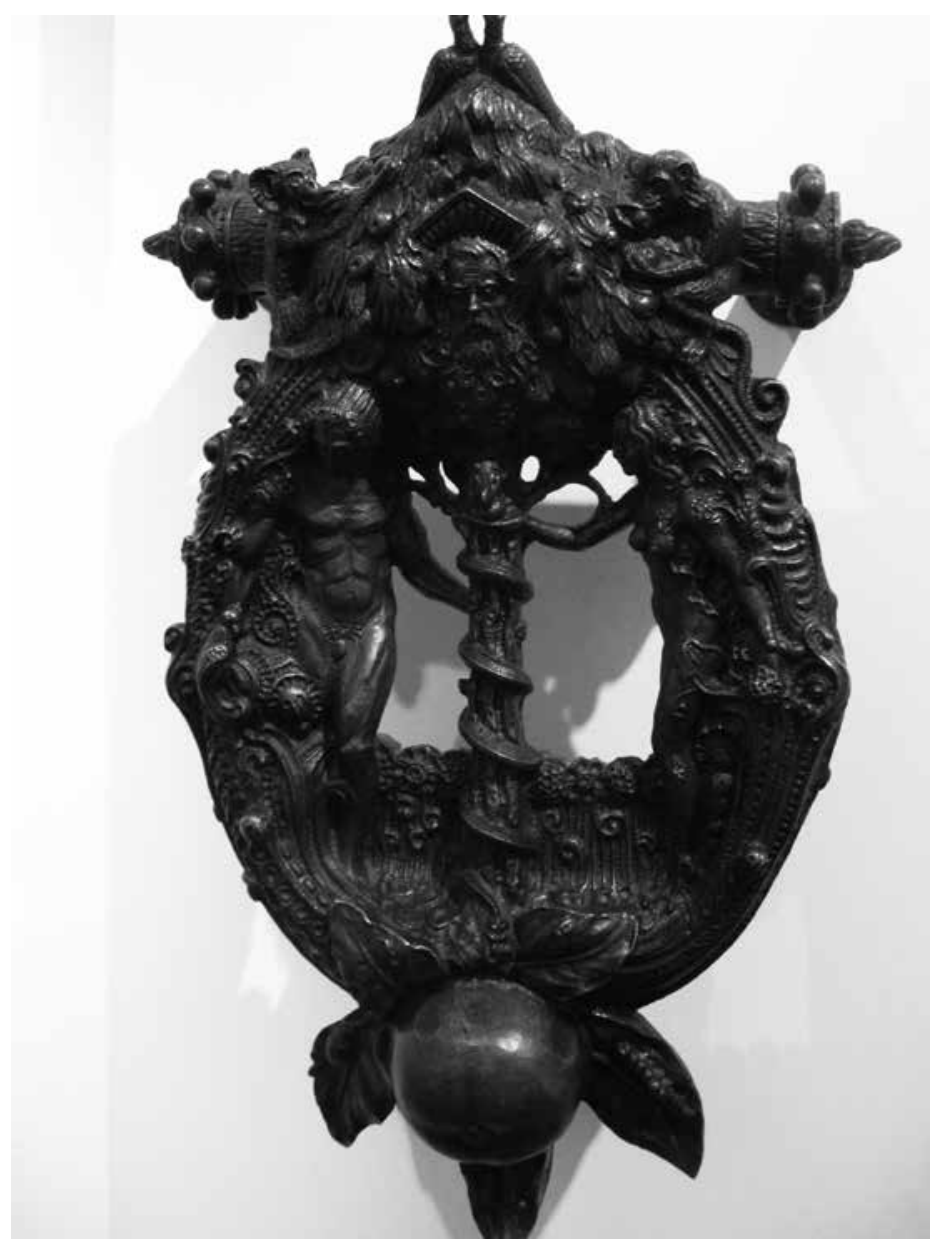

Fotografia: José Enrique Castillo 